\title{
Effect of an Exercise Bout Prior to the Booster Dose of an Inactivated SARS-CoV-2 Vaccine on Immunogenicity in Immunocompromised Patients
}

Bruno Gualano ( $\boldsymbol{\sigma}$ gualano@usp.br)

Universidade de São Paulo

Carla G. S. Saad

Universidade de Sao Paulo

Sofia M. Sieczkowska

Universidade de Sao Paulo

Ítalo Ribeiro Lemes

Universidade de Sao Paulo

Rafael Pires da Silva

Universidade de Sao Paulo

Ana J. Pinto

University of Colorado Anschutz Medical Campus

Bruna C. Mazzolani

Universidade de Sao Paulo

Fabiana I. Smaira

Universidade de Sao Paulo

Saulo Gil

Universidade de Sao Paulo

Gersiel Oliveira-Junior

Universidade de Sao Paulo

Nadia E. Aikawa

Universidade de Sao Paulo

Ana C. Medeiros-Ribeiro

Universidade de Sao Paulo

Clovis A. Silva

Universidade de Sao Paulo

Emily F. N. Yuki

Universidade de Sao Paulo

Sandra G Pasoto

Universidade de Sao Paulo

Rosa M.R. Pereira

Universidade de Sao Paulo 


\section{Samuel K. Shinjo}

Universidade de Sao Paulo

Danieli C. O. Andrade

Universidade de Sao Paulo

Percival D. Sampaio-Barros

Universidade de Sao Paulo

\section{Hamilton Roschel}

Universidade de Sao Paulo

\section{Eloisa Bonfa}

Universidade de Sao Paulo

\section{Short Report}

Keywords: COVID-19, physical activity, Sinovac-CoronaVac, immunosuppression

Posted Date: December 28th, 2021

DOI: https://doi.org/10.21203/rs.3.rs-1203127/v1

License: @ (i) This work is licensed under a Creative Commons Attribution 4.0 International License. Read Full License

Version of Record: A version of this preprint was published at Journal of Applied Physiology on February 10th, 2022. See the published version at https://doi.org/10.1152/japplphysiol.00015.2022. 


\section{Abstract}

This study aimed to investigate whether a single bout of exercise prior to the homologous booster dose of a SARS-CoV-2 inactivated vaccine (Sinovac-CoronaVac) could enhance immunogenicity in patients with dysfunctional immune system. This was a randomized controlled trial (1:1) within a single-arm, phase 4 vaccination trial, conducted in São Paulo, Brazil. Patients with spondyloarthritis assigned to the intervention group performed an exercise bout comprising three unilateral strength exercises involving eccentric and concentric contractions. After exercising, patients remained at rest for $1 \mathrm{~h}$ prior to vaccination, which was applied to the exercised arm. The control group remained at rest before vaccination. Immunogenicity was assessed before (Pre) and one month after (Post) the booster dose using seropositivity rates of total anti-SARSCoV-2 S1/S2 IgG, geometric mean titers of anti-S1/S2 IgG (GMT), frequency of NAb positivity, and NAb activity. Before the booster dose, 16 patients from the exercise group and 16 patients from the control group exhibited seropositivity for IgG (59\% vs. $57.1 \%)$, one month after the booster dose, seropositivity occurred in $96 \%$ vs. $100 \%$ of the cases $(p=0.84$, group by time interaction). Only 10 patients from the exercise group and 12 patients from the control group showed positive NAb serology at Pre (37\% vs. $42.8 \%)$. One month following the booster, NAb positivity was $96 \%$ vs. $93 \%$ ( $p=0.41$, group-by-time interaction). GMT was comparable between groups at Pre ( $p>0.05)$. At Post, GMT increased similarly in both groups (exercise: $56.9 \%$; control: $57.9 \%$ ), with no group-by-time interaction ( $p=0.82$; estimated mean difference between groups at Post [EMD]: $-40.4 \mathrm{UA} / \mathrm{mL}$, $95 \% \mathrm{Cl}:-327,246 \mathrm{UA} / \mathrm{mL}$ ). Likewise, NAb activity was similar between groups at Pre and increased similarly in both of them as a result of the booster ( $47.5 \%$ vs. $39.9 \%)$, with no group-by-time interaction ( $p=0.99$; EMD: $-6.19 \%, 95 \% \mathrm{Cl}:-17 ; 4.6 \%)$. In conclusion, a single bout of exercise did not enhance immunogenicity to a homologous booster dose of an inactivated SARS-CoV-2 vaccine among patients with spondyloarthritis. Studies assessing exercise as an adjuvant to first or second doses remain necessary.

\section{Highlights}

- We tested the role of exercise as an adjuvant to a booster of a COVID-19 vaccine

- Immunocompromised patients were immunized after an acute bout of exercise or not

- Patients exhibited an excellent immunogenicity in response to the booster dose

- Exercise did not add to the vaccine effects on IgG or neutralizing antibodies

- Whether exercise acts as an adjuvant to the first/second doses remains unknown

\section{Introduction}

Immunocompromised individuals generally exhibit lower immunogenicity to SARS-CoV vaccines (Aikawa et al., 2021; Furer et al., 2021; Geisen et al., 2021; Medeiros-Ribeiro et al., 2021). Regular exercise can enhance the responses to some vaccines (e.g., influenza) in individuals with dysfunctional immune system (Kohut et al., 2004; Rogers et al., 2008; Woods et al., 2009), an observation recently extended to a SARS-CoV-2 vaccine (Gualano et al., in press).

Of relevance, a single exercise session has been shown to be sufficient to enhance the humoral and cellular response to vaccines (Edwards et al., 2012, 2007). This immunoenhancement effect seems to be associated with the exercise-induced local inflammatory process, which involves the immediate secretion of cytokines 
(e.g., IL-6), edema, hyperemia, increased vascular permeability, and invasion of immune cells (Edwards et al., $2010,2007)$. However, to our knowledge, this hypothesis has not yet been tested in the context of SARS-CoV vaccines.

In this exploratory study within a phase 4 vaccination trial, we tested whether a single bout of exercise prior to the homologous booster dose of a SARS-CoV-2 inactivated vaccine (Sinovac-CoronaVac, the most frequently administered vaccine worldwide) could enhance immunogenicity in patients with spondyloarthritis (SpA), who were shown to experience suboptimal vaccine responses following a two-dose vaccination course (MedeirosRibeiro et al., 2021).

\section{Experimental Design And Methods}

\subsection{Participants}

This was a randomized controlled trial (1:1) within a single-arm, phase 4 vaccination trial, conducted in a tertiary hospital in Sao Paulo, Brazil. The main trial was designed to test the effectiveness and safety of Sinovac-CoronaVac in a large cohort of patients with systemic autoimmune rheumatic diseases (clinicaltrials.gov \#NCT04754698). This substudy was approved by the local ethics committee as an addendum to the main trial (CAAE: 42566621.0.0000.0068), and all patients agreed to participate by providing an informed consent. On the basis of feasibility, resources, capacity of research staff and facility, and patients' availability (Bacchetti, 2010; Bacchetti et al., 2008), we selected 60 consecutive patients with SpA (ankylosing spondylitis and psoriatic arthritis), diagnosed according to stablished criteria (Linden et al., 1984; Taylor et al., 2006). These patients had been invited to receive the homologous booster dose of Sinovac-CoronaVac (six months after the second dose) in the context of the main trial. Eligibility criteria were described elsewhere (Medeiros-Ribeiro et al., 2021). Specifically, for this trial, age < 50 years, current use of methotrexate, and any health condition precluding exercise were considered exclusion criteria. The randomization list for intervention (exercise) or control (no exercise) was created using a computer-generated code.

\subsection{Experimental design}

Patients assigned to the exercise group performed an exercise bout in our intrahospital exercise physiology laboratory, located at the same medical center where vaccination was administered. The exercise session was conducted by experienced trainers and was composed of a specific warm-up followed by three unilateral strength exercises using dumbbells (i.e., scapular abduction, elbow flexion and elbow extension) involving eccentric and concentric contractions. Four sets of 8-12 maximum repetitions (until concentric failure) were performed for each exercise, interspaced by a 30-s interval. The total duration of the exercise session was approximately $20 \mathrm{~min}$. After exercising, patients remained at rest for $1 \mathrm{~h}$ prior to vaccination (third dose of CoronaVac - Sinovac Life Sciences, Beijing, China), injected into the exercised arm. The control group remained at rest for $1 \mathrm{~h}$ before vaccination. All patients underwent either the exercise or control conditions and were vaccinated on a single day (September 18, 2021). As a proxy of exercise-induced local edema/inflammation, maximal circumference of the biceps at elbow flexion was measured before and after exercise using a measuring tape. In addition, muscle soreness of the exercised limb was assessed before and after 0, 24 and $48 \mathrm{~h}$ from the exercise session, using a visual analogue scale ranging from 0 (no pain) to 10 (worst possible pain). 


\subsection{Immunogenicity outcomes}

Blood samples $(20 \mathrm{~mL})$ were collected immediately before (Pre) and approximately 1 month after the third dose injection (Post) and were stored in a freezer at $-70^{\circ} \mathrm{C}$. Laboratory staff was blinded to the intervention. Immunogenicity was assessed using seroconversion rates of total anti-SARS-CoV-2 S1/S2 IgG (considering values $>15.0 \mathrm{UA} / \mathrm{mL}$ ), geometric mean titers of anti-S1/S2 IgG (GMT), frequency of positive NAb (inhibition $\geq$ $30 \%$ ), and NAb activity for the positive samples. GMT and NAb assays were thoroughly described elsewhere (Gualano et al., in press; Medeiros-Ribeiro et al., 2021).

\subsection{Statistical analysis}

Generalized Estimating Equations (GEE) models were used to assess possible differences between groups, assuming fixed effects for group and time, and random effects for patients. Post-hoc tests with Tukey's adjustment were performed for multiple pairwise comparisons purposes when necessary. Two exploratory analyses were conducted. First, sex, disease (ankylosing spondylitis and psoriatic arthritis) and age were used as covariates in GEE models. Second, GEE models were also used to assess possible differences between groups for GMT and NAb activity in an exploratory, sensitivity analysis involving only patients who did not exhibit seropositivity for IgG (exercise: $n=11$; control: $n=12$ ) or NAb (exercise: $n=17$; control: $n=16$ ) before the booster dose. Data are expressed as absolute and relative frequencies, or estimated mean difference between groups at Post (EMD) $\pm 95 \%$ confidence intervals $(95 \% \mathrm{CI})$, and relative delta change $(\%)$, excepted otherwise stated. Significance level was previously set at $p \leq 0.05$. Analyses were performed in RStudio version 4.02, using the following packages: "geepack", "stats”, "tydverse”, "emmeans", "ggplot2" and "ggpubr".

\section{Results}

Sixty patients were randomly assigned to either exercise or control groups ( $\mathrm{n}=30$ per group). Three patients in the exercise group did not receive the booster dose (due to personal reasons), whereas 2 patients in the control group did not attend the post-vaccination visit to collect blood sample. Therefore, 27 patients in the exercise group and 28 patients in the control group were included in the analyses. Patients' main characteristics are described in Table 1. 
Table 1

Main patients' characteristics in the exercise and control groups

\begin{tabular}{|c|c|c|}
\hline & Exercise $(n=27)$ & Control $(n=30)$ \\
\hline Age, years (mean $\pm S D$ ) & $58.8 \pm 5.3$ & $60.8 \pm 5.8$ \\
\hline Body mass index, $\mathrm{kg} / \mathrm{m}^{2}$ (mean $\left.\pm \mathrm{SD}\right)$ & $28.0 \pm 8.0$ & $27.1 \pm 4.4$ \\
\hline Disease duration, years (mean $\pm S D$ ) & $28.6 \pm 10.7$ & $28.5 \pm 11.0$ \\
\hline Sex, n (Male/Female) & $20 / 7$ & $20 / 8$ \\
\hline \multicolumn{3}{|l|}{ Disease n (\%) } \\
\hline Ankylosing spondylitis & $20(74)$ & $20(71.4)$ \\
\hline Psoriatic arthritis & $7(26)$ & $8(28.6)$ \\
\hline \multicolumn{3}{|l|}{ Treatment n (\%) } \\
\hline Biologic therapy* & $10(37.0)$ & $19(67.8)$ \\
\hline Immunosuppressants & $4(15.0)$ & $4(14.2)$ \\
\hline Prednisone & $4(15.0)$ & $1(3.5)$ \\
\hline \multicolumn{3}{|l|}{ Comorbidities n(\%) } \\
\hline Arterial hypertension & $20(74.0)$ & $14(50.0)$ \\
\hline Diabetes mellitus & $5(19.0)$ & $11(39.2)$ \\
\hline Chronic renal disease & $2(7.0)$ & $2(7.1)$ \\
\hline IgG positivity, n(\%) & $16(59.0)$ & $16(57.1)$ \\
\hline Nab positivity, n(\%) & $10(37.0)$ & $12(42.8)$ \\
\hline \multicolumn{3}{|c|}{$\begin{array}{l}\text { *Biologic therapy: TNF inhibitor (exercise } n=4 \text {; control } n=16) \text {, monoclonal antibodies (exercise } n=10 \text {; } \\
\text { control } n=17) \text {, etanercept (exercise } n=0 \text {; control } n=4) \text {, adalimumab (exercise } n=3 \text {; control } n=7 \text { ), } \\
\text { infliximab (exercise } n=1 \text {; control } n=5) \text {, certolizumab pegol (exercise } n=1 \text {; control } n=0) \text {; } \\
\text { Immunosuppressants: leflunomide (exercise } n=4 \text {; control } n=3 \text { ), cyclosporin (exercise } n=1 \text {; control } n=0) \text { ). } \\
\text { Note: Sums to more than the patient numbers provided because seven patients were taking more than one } \\
\text { biologic agent. }\end{array}$} \\
\hline
\end{tabular}

Biceps muscle circumference was increased immediately after exercise $(p<0.01)$. Likewise, muscle soreness was also increased across time (before: $1.1 \pm 2.1$; immediately after exercise: $2.6 \pm 2.8 ; 24$ h: $4.4 \pm 2.5 ; 48$ h: 3.5 \pm 2.5 ; all $p<0.01$ vs. pre-exercise). These data suggest that exercise effectively induced muscle edema/inflammation.

At Pre, 16 patients from the exercise group and 16 patients from the control group exhibited similar seropositivity rates for IgG ( $59 \%$ vs. $57.1 \%$, respectively). One month after the third dose, IgG seropositivity rates were comparable between groups (96\% vs. 100\%), with no group-by-time interaction ( $p=0.84)$.

As for NAb positivity, 10 patients from the exercise group and 12 patients from the control group showed positive serology (37\% vs. $42.8 \%$ ) at Pre. One month following the booster dose, positive serology was similar 
between the two groups (96\% vs. 93\%), with no group-by-time interaction $(p=0.41)$.

GMT was comparable between exercise and control groups at Pre ( $p$ > 0.05). After the booster dose, GMT increased similarly in both groups (56.9\% vs. 57.9), with no group-by-time interaction ( $p=0.82$; EMD: -40.4 $\mathrm{UA} / \mathrm{mL}, 95 \% \mathrm{Cl}:-327,246 \mathrm{UA} / \mathrm{mL})$. Likewise, NAb activity was similar between groups at Pre, and it increased similarly in both of them after the booster (47.5\% vs. $39.9 \%)$; there was no group-by-time interaction ( $p=0.99$; EMD: $-6.19 \%, 95 \% \mathrm{Cl}:-17 ; 4.6 \%$ ) (Figure 1). Models adjusted by sex, disease (ankylosing spondylitis and psoriatic arthritis) and age as covariates yielded similar results (data not shown).

In the sensitivity analysis involving only patients who exhibited no seropositivity for IgG and NAb at Pre, similar

increases in GMT (82.2\% vs. 92.6\%) and Nab activity (66.3\% vs. 66.5\%) were observed in both groups following the booster (main effect of time, $p<0.01$ for both), with no group-by-time interactions $(p=0.24$ and $p=0.50$ for GMT and Nab activity; EMD: $-110 \mathrm{UA} / \mathrm{mL}, 95 \% \mathrm{Cl}:-292,72.5 \mathrm{UA} / \mathrm{mL}$ and EMD: $-3.1 \%, 95 \% \mathrm{Cl}:-12.2,5.9 \%$, respectively).

\section{Discussion}

The search for strategies able to enhance SARS-CoV-2 vaccine responses is key to countering the pandemic, particularly for individuals with dysfunctional immune system. Growing evidence now shows that immunocompromised individuals respond less robustly to different SARS-CoV-2 vaccines (Medeiros-Ribeiro et al., 2021). This is the case for patients with SpA, which are immune-mediated inflammatory diseases characterized by axial and/or peripheral joint inflammation, as well as extra-articular manifestations. In the previous phase of this phase 4 trial, a two-dose course of Sinovac-CorovaVac (Medeiros-Ribeiro et al., 2021), a WHO-approved vaccine that protects against severe cases and mortality of COVID-19 (Jara et al., 2021), led to a moderate immunogenicity among immunocompromised patients (including those with SpA). In this substudy within the phase 4 trial, we speculated that an acute exercise session could enhance the responses of the booster dose in this group, a hypothesis that was refuted by the current findings.

There is evidence that physical activity improves immune response to vaccination, particularly in individuals at risk for immune dysfunction. For instance, studies involving older adults show increased responses to vaccination in physically active individuals vs. inactive ones, or in those who received an exercise intervention vs. non-exercised ones (Keylock et al., 2007; Kohut et al., 2004; Pascoe et al., 2014). Recently, we also showed that among a large cohort of patients with systemic autoimmune rheumatic diseases, those who were physically active exhibited higher levels of antibodies following two doses of Sinovac-CorovaVac when compared to inactive individuals (Gualano et al., in press). Next, we tested the immunoenhancement effect of an exercise session in a subgroup of this cohort (i.e., SpA), on the basis of evidence showing that a single bout of exercise at the time of vaccination can elicit better immune responses (Edwards et al., 2012). The reason underlying the null findings of the present study may rely in the fact that the immunoenhancement effect of exercise commonly manifests for antigens eliciting weak immune responses (Edwards et al., 2012; Pascoe et al., 2014; Ranadive et al., 2013). This notion is well illustrated by the findings showing that exercise was able to enhance the responses to a reduced dose of pneumococcal vaccine, but did not change the responses to the full dose (Edwards et al., 2012). In addition, a 45-min walk was shown to be immunostimulatory in older 
women who had lower pre-vaccine titers, but not in older men with stronger immune responses (Ranadive et al., 2013).

To test the hypothesis that patients with weaker immunity would primarily benefit from the immunoenhancement effect of exercise, we sub-analyzed the patients with no NAb or IgG seropositivity before the booster. The results indicated no effect of the intervention, corroborating the lack of immunostimulatory effects of exercise in this study. It is likely that the booster shot itself resulted in such a robust antibody response that masked any adjuvant role of exercise. In fact, one month following the booster dose, out of the 55 patients analyzed, only 1 did not exhibit IgG seropositivity, and 3 did not show NAb positivity. This excellent immunogenicity to the booster may have created a ceiling effect for exercise.

This study has limitations. First, the sample size was relatively low, despite the homogenous characteristics of the participants. Second, despite the fact that this study investigated diseases that represent the archetype of immune system dysfunction, the current findings cannot be promptly generalized to other conditions affecting the immune function. Third, as previously discussed, this three-dose schedule resulted in a strong immune response, which may have masked any enhancement effect of exercise. Studies assessing exercise as an adjuvant to first, second or even booster half-doses are still warranted. Finally, regular exercise may confer enhanced vaccine responses through distinct mechanisms from those of acute exercise (for a review, see Pascoe et al., 2014), so that the present findings are restricted to the latter model of intervention.

In conclusion, a single bout of exercise did not enhance the excellent immunogenicity in response to a homologous booster dose of an inactivated SARS-CoV-2 vaccine among immunocompromised patients with SpA.

\section{Declarations}

The authors have no conflict of interests.

\section{References}

1. Aikawa, N.E., Kupa, L.V.K., Pasoto, S.G., Medeiros-Ribeiro, A.C., Yuki, E.F.N., Saad, C.G.S., Pedrosa, T., Fuller, R., Shinjo, S.K., Sampaio-Barros, P.D., Andrade, D.C.O., Pereira, R.M.R., Seguro, L.P.C., Valim, J.M.L., Waridel, F., Sartori, A.M.C., Duarte, A.J.S., Antonangelo, L., Sabino, E.C., Menezes, P.R., Kallas, E.G., Silva, C.A., Bonfa, E., 2021. Immunogenicity and safety of two doses of the CoronaVac SARS-CoV-2 vaccine in SARS-CoV-2 seropositive and seronegative patients with autoimmune rheumatic diseases in Brazil: a subgroup analysis of a phase 4 prospective study. Lancet Rheumatol. 1-12. https://doi.org/10.1016/s26659913(21)00327-1

2. Bacchetti, P., 2010. Current sample size conventions: Flaws, harms, and alternatives. BMC Med. https://doi.org/10.1186/1741-7015-8-17

3. Bacchetti, P., McCulloch, C.E., Segal, M.R., 2008. Simple, defensible sample sizes based on cost efficiency. Biometrics. https://doi.org/10.1111/j.1541-0420.2008.01004_1.x

4. Edwards, K.M., Burns, V.E., Allen, L.M., McPhee, J.S., Bosch, J.A., Carroll, D., Drayson, M., Ring, C., 2007. Eccentric exercise as an adjuvant to influenza vaccination in humans. Brain. Behav. Immun. 21, 209-217. 
https://doi.org/10.1016/J.BBI.2006.04.158

5. Edwards, K.M., Campbell, J.P., Ring, C., Drayson, M.T., Bosch, J.A., Downes, C., Long, J.E., Lumb, J.A., Merry, A., Paine, N.J., Burns, V.E., 2010. Exercise intensity does not influence the efficacy of eccentric exercise as a behavioural adjuvant to vaccination. Brain. Behav. Immun. 24, 623-630.

https://doi.org/10.1016/J.BBI.2010.01.009

6. Edwards, K.M., Pung, M.A., Tomfohr, L.M., Ziegler, M.G., Campbell, J.P., Drayson, M.T., Mills, P.J., 2012. Acute exercise enhancement of pneumococcal vaccination response: A randomised controlled trial of weaker and stronger immune response. Vaccine 30, 6389-6395.

https://doi.org/10.1016/J.VACCINE.2012.08.022

7. Furer, V., Eviatar, T., Zisman, D., Peleg, H., Paran, D., Levartovsky, D., Zisapel, M., Elalouf, O., Kaufman, I., Meidan, R., Broyde, A., Polachek, A., Wollman, J., Litinsky, I., Meridor, K., Nochomovitz, H., Silberman, A., Rosenberg, D., Feld, J., Haddad, A., Gazzit, T., Elias, M., Higazi, N., Kharouf, F., Shefer, G., Sharon, O., Pel, S., Nevo, S., Elkayam, O., 2021. Immunogenicity and safety of the BNT162b2 mRNA COVID-19 vaccine in adult patients with autoimmune inflammatory rheumatic diseases and in the general population: a multicentre study. Ann. Rheum. Dis. 80, 1330-1338. https://doi.org/10.1136/annrheumdis-2021-220647

8. Geisen, U.M., Berner, D.K., Tran, F., Sümbül, M., Vullriede, L., Ciripoi, M., Reid, H.M., Schaffarzyk, A., Longardt, A.C., Franzenburg, J., Hoff, P., Schirmer, J.H., Zeuner, R., Friedrichs, A., Steinbach, A., Knies, C., Markewitz, R.D.H., Morrison, P.J., Gerdes, S., Schreiber, S., Hoyer, B.F., 2021. Immunogenicity and safety of anti-SARSCoV-2 mRNA vaccines in patients with chronic inflammatory conditions and immunosuppressive therapy in a monocentric cohort. Ann. Rheum. Dis. 80, 1306-1311. https://doi.org/10.1136/ANNRHEUMDIS-2021220272

9. Gualano, B., Silva, R., Pinto, A., Mazzolani, B., Smaira, F., Sieczkowska, S., Aikawa, N., Pasoto, S., MedeirosRibeiro, A., Saad, C., Yuk, E., Silva, C., Swinton, P., Hallal, P., n.d. Physical activity associates with enhanced immunogenicity of an inactivated virus vaccine against SARS-CoV-2 in patients with autoimmune rheumatic diseases. Brain Behav. Immun.

10. Jara, A., Undurraga, E.A., González, C., Paredes, F., Fontecilla, T., Jara, G., Pizarro, A., Acevedo, J., Leo, K., Leon, F., Sans, C., Leighton, P., Suárez, P., García-Escorza, H., Araos, R., 2021. Effectiveness of an Inactivated SARS-CoV-2 Vaccine in Chile. N. Engl. J. Med. 385, 875-884. https://doi.org/10.1056/NEJMoa2107715

11. Keylock, K.T., Lowder, T., Leifheit, K.A., Cook, M., Mariani, R.A., Ross, K., Kim, K., Chapman-Novakofski, K., McAuley, E., Woods, J.A., 2007. Higher antibody, but not cell-mediated, responses to vaccination in high physically fit elderly. J. Appl. Physiol. 102, 1090-1098.

https://doi.org/10.1152/JAPPLPHYSIOL.00790.2006/ASSET/IMAGES/LARGE/ZDG0030770660005.JPEG

12. Kohut, M.L., Arntson, B.A., Lee, W., Rozeboom, K., Yoon, K.J., Cunnick, J.E., McElhaney, J., 2004. Moderate exercise improves antibody response to influenza immunization in older adults. Vaccine 22, 2298-2306. https://doi.org/10.1016/J.VACCINE.2003.11.023

13. Linden, S. Van Der, Valkenburg, H.A., Cats, A., 1984. Evaluation of Diagnostic Criteria for Ankylosing Spondylitis. Arthritis Rheum. 27, 361-368. https://doi.org/10.1002/art.1780270401

14. Medeiros-Ribeiro, A.C., Aikawa, N.E., Saad, C.G.S., Yuki, E.F.N., Pedrosa, T., Fusco, S.R.G., Rojo, P.T., Pereira, R.M.R., Shinjo, S.K., Andrade, D.C.O., Sampaio-Barros, P.D., Ribeiro, C.T., Deveza, G.B.H., Martins, V.A.O., Silva, C.A., Lopes, M.H., Duarte, A.J.S., Antonangelo, L., Sabino, E.C., Kallas, E.G., Pasoto, S.G., Bonfa, E., 
2021. Immunogenicity and safety of the CoronaVac inactivated vaccine in patients with autoimmune rheumatic diseases: a phase 4 trial. Nat. Med. 27, 1744-1751. https://doi.org/10.1038/s41591-021-014695

15. Pascoe, A.R., Fiatarone Singh, M.A., Edwards, K.M., 2014. The effects of exercise on vaccination responses: A review of chronic and acute exercise interventions in humans. Brain. Behav. Immun. 39, 33-41. https://doi.org/10.1016/j.bbi.2013.10.003

16. Ranadive, S.M., Cook, M., Kappus, R.M., Yan, H., Lane, A.D., Woods, J.A., Wilund, K.R., Iwamoto, G., Vanar, V., Tandon, R., Fernhall, B., 2013. Effect of acute aerobic exercise on vaccine efficacy in older adults. Med. Sci. Sports Exerc. 46, 455-461. https://doi.org/10.1249/MSS.0B013E3182A75FF2

17. Rogers, C.J., Zaharoff, D.A., Hance, K.W., Perkins, S.N., Hursting, S.D., Schlom, J., Greiner, J.W., 2008. Exercise enhances vaccine-induced antigen-specific T cell responses. Vaccine 26, 5407-5415. https://doi.org/10.1016/J.VACCINE.2008.07.081

18. Taylor, W., Gladman, D., Helliwell, P., Marchesoni, A., Mease, P., Mielants, H., 2006. Classification criteria for psoriatic arthritis: Development of new criteria from a large international study. Arthritis Rheum. 54, 26652673. https://doi.org/10.1002/art.21972

19. Woods, J.A., Keylock, K.T., Lowder, T., Vieira, V.J., Zelkovich, W., Dumich, S., Colantuano, K., Lyons, K., Leifheit, K., Cook, M., Chapman-Novakofski, K., McAuley, E., 2009. Cardiovascular Exercise Training Extends Influenza Vaccine Seroprotection in Sedentary Older Adults: The Immune Function Intervention Trial. J. Am. Geriatr. Soc. 57, 2183-2191. https://doi.org/10.1111/J.1532-5415.2009.02563.X

\section{Figures}



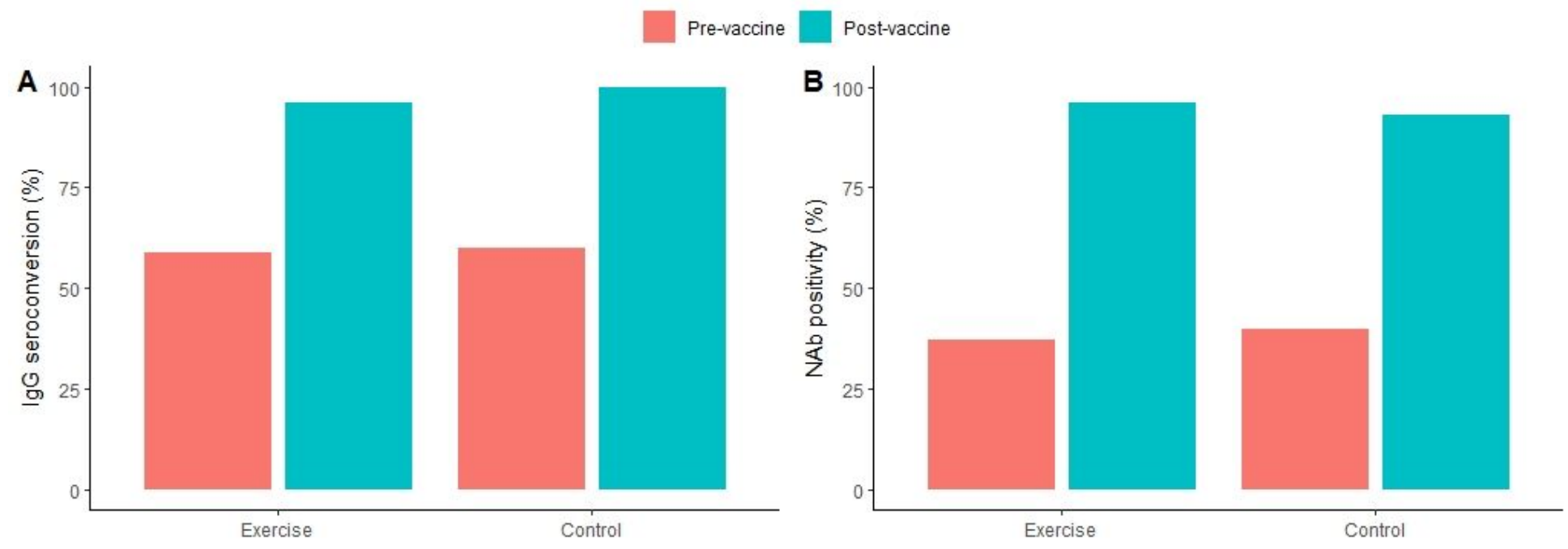

C

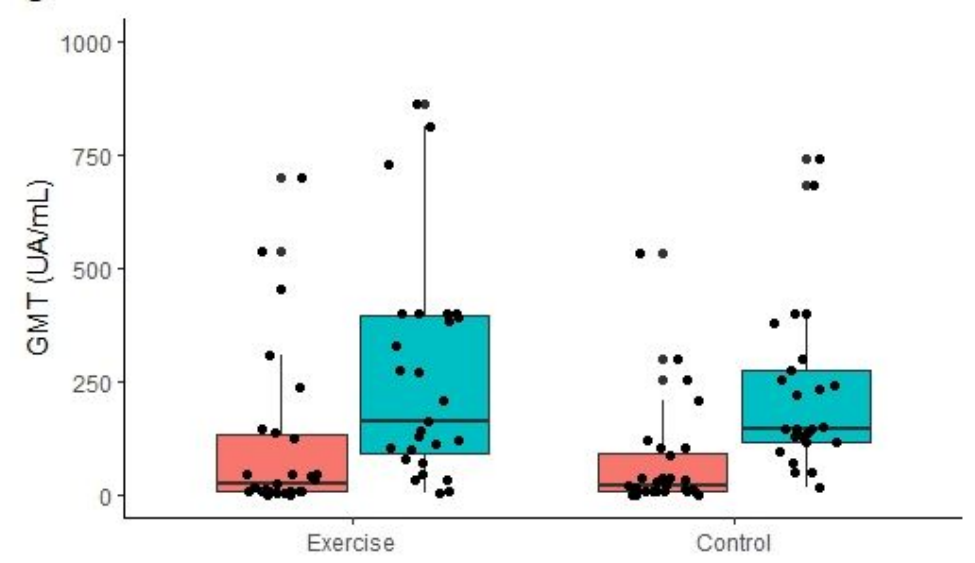

D

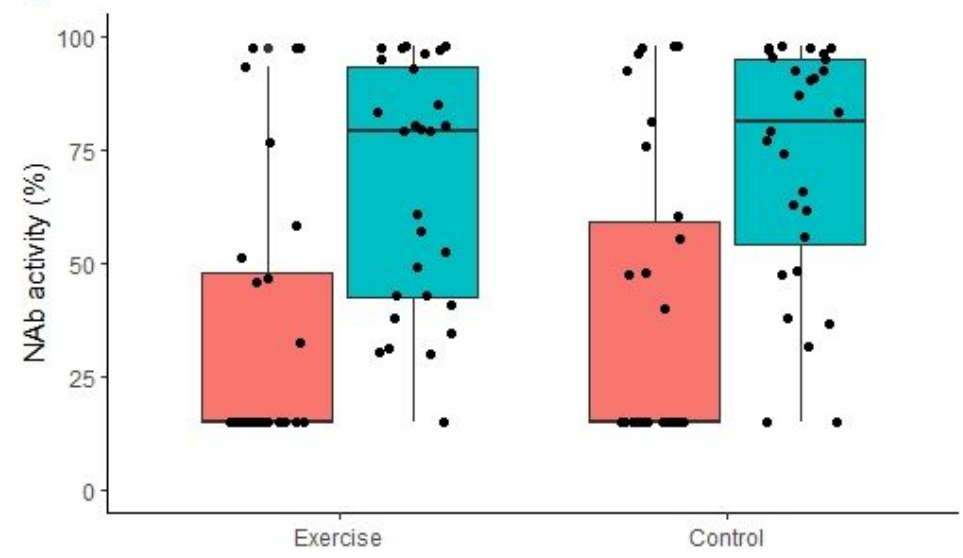

Figure 1

Panel A: IgG positivity rates were comparable between groups before (Pre) and increased similarly in both of them after the booster (group-by-time interaction, $p=0.84$ ). Panel $B$ : Nab positivity rates were also comparable between groups at Pre, and it increased similarly in both of them after the booster (group by time interaction, $\mathrm{p}$ $=0.41$ ). Panel C: geometric mean titers of anti-S1/S2 IgG (GMT) were comparable between groups at Pre, and increased similarly in both of them as a result of the booster (exercise: $56.9 \%$; control: $57.9 \%$ ), with no group-bytime interaction (EMD: $-40.4 \mathrm{UA} / \mathrm{mL}, 95 \% \mathrm{Cl}:-327,246 \mathrm{UA} / \mathrm{mL}, \mathrm{p}=0.82$ ). Panel $\mathrm{D}$ : NAb activity was similar between groups at Pre and increased similarly in both of them after the booster (exercise: $47.5 \%$, control: $39.9 \%$ ), with no group-by-time interaction (EMD: $-6.19 \%, 95 \% \mathrm{Cl}:-17 ; 4.6 \%, \mathrm{p}=0.99)$. Data were analyzed using GEE. Boxplot are expressed as median \pm interquartile (minimum; maximum).

\section{Supplementary Files}

This is a list of supplementary files associated with this preprint. Click to download.

- CONSORT2010FlowDiagramvaccine.pdf 